\title{
Consumption of Seafood, Serum Liver Enzymes, and Blood Levels of PFOS and PFOA in the Japanese Population
}

\author{
Miwa Yamaguchi ${ }^{1}$, Kokichi Arisawa ${ }^{1}$, Hirokazu UemurA ${ }^{1}$, \\ Sakurako KatsuUra-Kamano $^{1}$, Hidenobu TaKami ${ }^{1}$, Fusakazu SawachiKA ${ }^{1}$, \\ Mariko NaкAmoto ${ }^{1,2}$, Tomoya Juta ${ }^{1}$, Eisaku TodA ${ }^{3}$, Kei Mori ${ }^{3}$, Manabu Hasegawa ${ }^{3}$, \\ Masaharu TAnto ${ }^{3}$, Masayuki Shima ${ }^{4}$, Yoshio SumiYoshi ${ }^{4}$, Kazunori Kodama ${ }^{4}$, \\ Takaichiro SuZUKI ${ }^{4}$, Masaki NAGAI ${ }^{4}$ and Hiroshi SATOH ${ }^{4}$
}

\begin{abstract}
${ }^{1}$ Department of Preventive Medicine, Institute of Health Biosciences, the University of Tokushima Graduate School, Japan, ${ }^{2}$ Department of Public Health and Applied Nutrition, Institute of Health Biosciences, the University of Tokushima Graduate School, Japan, ${ }^{3}$ Ministry of the Environment of Japan and ${ }^{4}$ The Study Group of the Accumulation of Dioxins in Humans, Japan
\end{abstract}

\begin{abstract}
Consumption of Seafood, Serum Liver Enzymes, and Blood Levels of PFOS and PFOA in the Japanese Population: Miwa Yamaguchl, et al. Department of Preventive Medicine, Institute of Health Biosciences, the University of Tokushima Graduate School, Japan-Objective: Perfluorooctanesulfonate (PFOS) and perfluorooctanoate (PFOA) have been shown to accumulate in the human body. The purpose of the present study was to examine the factors associated with the blood levels of PFOS and PFOA. Methods: A cross-sectional study was performed on 307 men and 301 women (aged 16-76 years) living in 15 prefectures in Japan. Blood levels of PFOS and PFOA were measured by liquid chromatography-mass spectrometry. Hepatic enzymes ( $y$-GTP, GOT, and GPT) and $\omega-3$ polyunsaturated fatty acids (DHA and EPA) levels in serum were also measured. Associations between the levels of PFOS and PFOA in blood and the intake frequency of 41 kinds of dishes, foods and beverages and the serum levels of liver enzymes and $\omega-3$ polyunsaturated fatty acids were examined using rank correlations. Results: Frequency of intake of boiled fish in broth, sliced raw fish and coastal fish showed significant positive correlations with PFOS concentrations in blood after adjustments for potential confounders. Serum levels of GOT, GPT, DHA and EPA showed significant positive correlations with PFOS and PFOA in blood. There was also a significant regional difference in the blood levels of PFOS and
\end{abstract}

Received Nov 20, 2012; Accepted Mar 15, 2013

Published online in J-STAGE Apr 9, 2013

Correspondence to: K. Arisawa, Department of Preventive Medicine, Institute of Health Biosciences, the University of Tokushima Graduate School, 3-18-15, Kuramoto-cho, Tokushima 770-8503, Japan. (e-mail: karisawa@basic.med.tokushima-u.ac.jp)
PFOA, with medians being highest in the Tokai/Hokuriku/ Kinki region. Conclusions: These findings suggest that the concentrations of PFOS in blood were mainly associated with fish consumption and that the levels of PFOS and PFOA were associated with the serum levels of liver enzymes in Japanese populations. Further investigations are required to clarify the reason for the regional differences in blood levels of PFOS and PFOA in Japan.

(J Occup Health 2013; 55: 184-194)

Key words: Blood, Fish intake, Perfluorooctanesulfonate (PFOS), Perfluorooctanoate (PFOA), Serum liver enzymes

Perfluorochemicals (PFCs) are man-made chemical substances containing $\mathrm{C}-\mathrm{C}$ and $\mathrm{C}-\mathrm{F}$ bonds. PFCs are extremely resistant to degradation, and accumulate in the environment. Perfluorooctanesulfonate (PFOS) and perfluorooctanoate (PFOA) are two of the main PFCs. PFOS and PFOA have both hydrophilic and lipophilic properties. PFOS bioaccumulates in animal tissues through the food $\mathrm{web}^{1)}$, and exposure to PFOA occurs in part through drinking water and beverages ${ }^{2}$. The geometric mean elimination half-lives of PFOS and PFOA from human serum are approximately 4.8 and 3.5 years, respectively ${ }^{3)}$.

Recently, it has been reported that PFOS and PFOA have toxic effects on wildlife and humans. The toxicity includes neurobehavioral defects ${ }^{4}$, hepatotoxicity ${ }^{5)}$, immune toxicity and carcinogenicity ${ }^{6}$. A reduction in human exposure to PFCs has been reported following the phasing-out of perfluorosulfonyl-based compounds and the Environmental Protection Agency's program 
to reduce PFOA emissions and content in products ${ }^{7)}$. Decreasing levels of PFOS and PFOA have been observed in biomonitoring studies in the United States as well ${ }^{8)}$.

Dietary habits may play a crucial role in the accumulation of PFOS and PFOA in the human body. However, there is still debate over the sources of dietary exposure to PFOS and PFOA in humans. Several reports have indicated that PFOS and PFOA were transferred into the human body mainly through fish intake ${ }^{9,10)}$. On the other hand, research in Denmark showed positive associations between concentrations of PFOS in blood and consumption of red meat, animal fat and snacks, but not fish ${ }^{11)}$.

Few studies have investigated the factors associated with blood levels of PFOS and PFOA in Japan. Therefore, we intended to clarify the factors correlated with blood levels of PFOS and PFOA, especially with regard to sex, age, residential area, lifestyle, reproductive history, and blood biochemical tests, in the Japanese population.

\section{Materials and Methods}

Study subjects

The study subjects were participants in the "Survey on the Accumulation of Dioxins and Other Chemical Compounds in Humans" project, which has been performed under the supervision of the Japanese Ministry of the Environment since 2002. The purpose of this project is to collect data on blood levels and the dietary intake of dioxins and other chemical substances and their determinants in Japanese populations without occupational exposure ${ }^{12,13)}$. The surveys were carried out in five regional blocks: Hokkaido/Tohoku, Kanto/Koshin-etsu, Tokai/Hokuriku/ Kinki, Chugoku/Shikoku, and Kyushu/Okinawa. In 2008, one prefecture was selected from each of the five regional blocks, and from each prefecture, three study areas (urban, agricultural and fishing areas) were selected. Urban, agricultural and fishing areas represent the places where industries of commerce and manufacturing, farming and fishing take place, respectively. In each prefecture, 50 subjects, 20 from the urban area and 15 from each of the agricultural and fishing areas, were recruited. In 2009 and 2010, two study areas (an urban area and an agricultural or fishing area) were selected from each prefecture, and 35 subjects, 20 from the urban area and 15 from the agricultural or fishing area, were recruited. The number of study subjects was reduced in 2009 and 2010 because of financial constraints. Participants were recruited through magazines, posters, the website of a local government office, and broadcasts in the area. Subjects were eligible if they were 15-76 years old, had lived for at last 10 years without frequently leaving the residential area for work or other reasons and were not suffering from severe anemia. The project collected data on blood levels of PFOS and PFOA from 608 subjects (307 men and 301 women) during 2008-2010. Written informed consent was obtained from each participant. The study protocol was reviewed and approved by the Ethical Committee of the Ministry of the Environment of Japan.

\section{Questionnaire}

Participants were requested to complete a questionnaire that included questions on residential and occupational histories, history of previous diseases and treatments, smoking habits, and dietary habits. Regarding dietary habits, the subjects were asked how often they had consumed 41 dishes, foods, food groups and beverages over the previous month. For rice, the amount consumed (how many cups/day) was asked. For the other 40 dishes, foods and beverages, the frequency of intake was classified into 5 categories: rarely, 1-2 times month ${ }^{-1}, 1-2$ times week ${ }^{-1}$, 3-4 times week ${ }^{-1}$ and almost every day, which were converted to times week $^{-1}$, i.e., $0.1,0.35,1.5,3.5$ and 6 times week $^{-1}$, respectively. We did not specify the unit or portion of the foods consumed.

\section{Collection and analysis of blood samples}

Blood samples were collected in $10-\mathrm{m} l$ Vacutainer tubes containing sodium-heparin solution (Terumo VT-100 or Becton, Dickinson and Company 367677). PFOS and PFOA were extracted using a method previously described by Hansen et al. ${ }^{14)}$, with some modifications. Briefly, $0.5 \mathrm{ml}$ of blood, $1 \mathrm{~m} l$ of $0.5 \mathrm{M}$ tetrabutylammonium hydrogen sulfate solution, $2 \mathrm{ml}$ of $0.25 \mathrm{M}$ sodium carbonate buffer, $10 \mu l$ of $500 \mathrm{ng} \mathrm{m} l^{-1}$ PFOS- ${ }^{13} \mathrm{C}_{4}$ and $10 \mu l$ of $500 \mathrm{ng} \mathrm{ml} l^{-1}$ PFOA- ${ }^{13} \mathrm{C}_{4}$ were added to a tube and mixed. Following addition of $5 \mathrm{ml}$ of metyl tert-butyl ether (MTBE) to the solution, the organic and aqueous layers were separated by shaking and centrifugation, and the organic layer was removed and retained. The raffinate was rinsed with MTBE and separated. The organic layer of the first and the second extractions were combined, and the extract was evaporated and dissolved with $1 \mathrm{~m} l$ of acetonitrile. Then, the sample was passed into a cartridge column (Oasis MCX Vac Cartridge [3 cc, $60 \mathrm{mg}$, Waters], and eluted with $10 \mathrm{ml}$ of acetonitrile. The sample was further evaporated, reconstituted in $1 \mathrm{~m} l$ of methanol and passed through a filter to remove suspended materials and insoluble particles. PFOS and PFOA concentrations were determined using liquid chromatography (Agilent 1100, Agilent Technologies, Palo Alto, CA, U.S.A.)-mass spectrometry (API 4000 QTrap, AB Sciex, Framingham, MA, U.S.A.). The limit of quantification was $0.20 \mathrm{ng} \mathrm{m} l^{-1}$ 
for PFOS and $0.32 \mathrm{ng} \mathrm{m} l^{-1}$ for PFOA. In the recovery test $($ No. $=7$ ), percentage recovery was $95.6 \%$ (coefficient of variation $[\mathrm{C} . \mathrm{V}]=.1.7 \%$ ) for PFOS and $100.9 \%$ (C.V.=2.1\%) for PFOA. In this study, whole blood was used for analysis of PFOS and PFOA because not only the serum fraction but also red blood cells contain PFOS and PFOA.

Blood samples were also collected into 9-m $l$ Vacutainer tubes and used for the measurement of $\gamma$-glutamyl transpeptidase $(\gamma$-GTP), glutamic-oxaloacetic transaminase (GOT), glutamic-pyruvic transaminase (GPT), docosahexaenoic acid (DHA) and eicosapentaenoic acid (EPA). Liver enzymes and $\omega-3$ polyunsaturated fatty acids in serum were determined by an automatic biochemical analyzer (Hitachi 7450, Japan) and by a gas chromatograph (Agilent Technologies 6890N, U.S.A), respectively, at SRL, Inc., (Tokyo, Japan).

\section{Statistical analysis}

The statistical significance of differences in blood levels of PFOS and PFOA according to sex, age category, regional block, residential area, smoking habits, reproductive history and method of nursing in women was tested using general linear models, adjusted for age, sex, log (BMI), regional block, residential area and smoking habits, excluding the independent variable of interest. In this analysis, the levels of PFOS and PFOA in blood were log-transformed because they were positively skewed. The antilog values of least-square means, adjusted for other factors included in the models, are presented. Associations between the concentrations of PFOS and PFOA in blood and the frequency of intake of dihses, foods and alcoholic beverages and the serum levels of liver enzymes and $\omega-3$ polyunsaturated fatty acids were examined using the Spearman rank correlation, after adjusting for the effects of age, sex, BMI, regional block, and smoking habits (Model 1). Using items that showed significant correlations in Model 1, fully adjusted analyses were performed for dishes, and foods and alcoholic beverages, separately (Model 2). In the analysis of liver enzymes, frequency of intake of alcoholic beverages was further adjusted (Model 2). In multivariate analysis, indicator variables were created for sex (No.=2), regional block (No.=5), smoking habits (No.=3), and frequency of alcohol drinking (tertiles, No.=3), and all categories except for the reference categories were included in the models. We performed all statistical analyses using the GLM and CORR functions of the SAS software package (version 8.2; SAS Institute Inc. 1997) ${ }^{15)}$. Results were considered statistically significant if $p$-values were less than 0.05 (two-tailed).

\section{Results}

Table 1 shows the characteristics of the study population. The proportions of men and women were 50.5 and $49.5 \%$, respectively, and the mean age was 46.3 years (S.D. 13.2 years). Because of the study design, the participants were distributed almost equally according to regional block. The proportion of subjects from urban areas comprised 44.2\%, followed by fishing villages $(33.6 \%)$ and farming villages $(22.2 \%)$. Table 1 also shows the proportion of nonsmokers, current smokers and ex-smokers, according to sex.

Table 2 presents the medians (with 25 th and 75 th

Table 1. Characteristics of the study population

\begin{tabular}{|c|c|c|}
\hline & No. & $(\%)$ \\
\hline Total & 608 & 100 \\
\hline \multicolumn{3}{|l|}{ Sex } \\
\hline Men & 307 & 50.5 \\
\hline Women & 301 & 49.5 \\
\hline \multicolumn{3}{|l|}{ Age } \\
\hline $16-29$ & 71 & 11.7 \\
\hline 30-39 & 134 & 22.0 \\
\hline $40-49$ & 141 & 23.2 \\
\hline $50-59$ & 151 & 24.8 \\
\hline $60-76$ & 111 & 18.3 \\
\hline \multicolumn{3}{|l|}{ Survey year } \\
\hline 2008 & 257 & 42.3 \\
\hline 2009 & 178 & 29.3 \\
\hline 2010 & 173 & 28.5 \\
\hline \multicolumn{3}{|l|}{ Regional block } \\
\hline Hokkaido/Tohoku & 123 & 20.2 \\
\hline Kanto/Koshin-estu & 122 & 20.1 \\
\hline Tokai/Hokuriku/Kinki & 127 & 20.9 \\
\hline Chugoku/Shikoku & 120 & 19.7 \\
\hline Kyushu/Okinawa & 116 & 19.1 \\
\hline \multicolumn{3}{|l|}{ Residential area } \\
\hline Urban & 269 & 44.2 \\
\hline Farming villages & 135 & 22.2 \\
\hline Fishing villages & 204 & 33.6 \\
\hline \multicolumn{3}{|l|}{ Smoking habits (men) } \\
\hline Nonsmoker & 117 & 38.1 \\
\hline Current smoker & 94 & 30.6 \\
\hline Ex-smoker & 96 & 31.3 \\
\hline \multicolumn{3}{|l|}{ Smoking habits (women) } \\
\hline Nonsmoker & 265 & 88.0 \\
\hline Current smoker & 20 & 6.6 \\
\hline Ex-smoker & 13 & 4.3 \\
\hline Unknown & 3 & 1.0 \\
\hline
\end{tabular}


Table 2. PFOS and PFOA concentrations in blood, $\omega$-3 polyunsaturated fatty acid and hepatic enzymes levels in serum, body mass index, and frequency of intake of dishes, foods and alcoholic beverages

\begin{tabular}{|c|c|c|c|c|}
\hline & No. & Median & 25 percentile & 75 percentile \\
\hline PFOS (ng/ml) & 608 & 5.8 & 3.7 & 8.8 \\
\hline PFOA (ng/ml) & 608 & 2.1 & 1.5 & 3.3 \\
\hline DHA (mg/l) & 608 & 127 & 97 & 162 \\
\hline EPA $(\mathrm{mg} / \mathrm{l})$ & 608 & 55 & 33 & 83 \\
\hline$\gamma-\mathrm{GTP}(\mathrm{IU} / l)$ & 608 & 23 & 16 & 40 \\
\hline GOT (IU/l) & 608 & 20 & 17 & 25 \\
\hline GPT (IU/l) & 608 & 19 & 14 & 28 \\
\hline BMI $\left(\mathrm{kg} / \mathrm{m}^{2}\right)$ & 608 & 22.9 & 20.9 & 25.5 \\
\hline \multicolumn{5}{|l|}{ Frequency of food intake } \\
\hline \multicolumn{5}{|l|}{ Dishes (times/week) } \\
\hline Grilled fish & 608 & 1.5 & 0.35 & 3.5 \\
\hline Eel & 601 & 0.1 & 0.1 & 0.1 \\
\hline Boiled fish in broth & 605 & 0.35 & 0.35 & 1.5 \\
\hline Deep-fried seafood or vegetables (tempura) & 606 & 0.35 & 0.35 & 0.35 \\
\hline Sliced raw fish (sashimi) & 608 & 0.35 & 0.35 & 1.5 \\
\hline Chinese-style dumpling (gyoza) & 606 & 0.35 & 0.35 & 0.35 \\
\hline Pork cutlets & 606 & 0.35 & 0.35 & 0.35 \\
\hline Grilled meat & 607 & 0.35 & 0.35 & 0.35 \\
\hline Hamburger & 607 & 0.1 & 0.1 & 0.35 \\
\hline Chicken nuggets & 607 & 0.1 & 0.1 & 0.35 \\
\hline Croquette & 608 & 0.35 & 0.35 & 0.35 \\
\hline French fries & 606 & 0.1 & 0.1 & 0.35 \\
\hline Chinese noodles & 608 & 0.35 & 0.35 & 1.5 \\
\hline Rice (bowls/day) & 608 & 2.5 & 2.0 & 3.0 \\
\hline \multicolumn{5}{|l|}{ Food items (times/week) } \\
\hline Coastal fishes (horse mackerel, mackerel, sardine, etc.) & 606 & 1.5 & 0.35 & 1.5 \\
\hline Other fishes (tuna, salmon, bonito, etc.) & 608 & 1.5 & 0.35 & 1.5 \\
\hline Squid, octopus & 606 & 0.35 & 0.35 & 0.35 \\
\hline Crab & 605 & 0.1 & 0.1 & 0.35 \\
\hline Shrimp & 604 & 0.35 & 0.1 & 0.35 \\
\hline Boiled fish paste, tubular roll of grilled fish paste (kamaboko, chikuwa) & 606 & 0.35 & 0.35 & 1.5 \\
\hline Short-neck clam, corbicula & 606 & 0.35 & 0.1 & 0.35 \\
\hline Beef & 606 & 1.5 & 0.35 & 1.5 \\
\hline Pork & 607 & 1.5 & 1.5 & 3.5 \\
\hline Ham, sausage & 607 & 1.5 & 0.35 & 1.5 \\
\hline Bacon & 604 & 0.35 & 0.1 & 1.5 \\
\hline Egg & 606 & 3.5 & 1.5 & 6.0 \\
\hline Milk & 607 & 1.5 & 0.35 & 6.0 \\
\hline Cheese & 607 & 0.35 & 0.1 & 1.5 \\
\hline Yogurt & 608 & 1.5 & 0.35 & 3.5 \\
\hline Butter & 603 & 0.35 & 0.1 & 1.5 \\
\hline Green-yellow leafy vegetables (spinach, komatsuna, etc.) & 605 & 1.5 & 1.5 & 3.5 \\
\hline Other green-yellow vegetables (carrot, pumpkin, etc.) & 607 & 3.5 & 1.5 & 6.0 \\
\hline Beans & 607 & 1.5 & 1.5 & 3.5 \\
\hline Sea tangle, seaweed & 607 & 1.5 & 1.5 & 3.5 \\
\hline Mushrooms & 608 & 1.5 & 1.5 & 3.5 \\
\hline Fruits & 608 & 3.5 & 1.5 & 6.0 \\
\hline Vegetable oil & 602 & 3.5 & 1.5 & 6.0 \\
\hline Margarine & 605 & 0.35 & 0.1 & 1.5 \\
\hline Beer & 603 & 0.35 & 0.1 & 3.5 \\
\hline Rice wine (sake) & 598 & 0.1 & 0.1 & 0.1 \\
\hline Spirits (syochu) & 599 & 0.1 & 0.1 & 0.35 \\
\hline Alcoholic beverages (beer, sake, syochu) & 593 & 0.55 & 0.3 & 5.35 \\
\hline
\end{tabular}

PFOS, Perfluorooctanesulfonate; PFOA, perfluorooctanoate; DHA, docosahexaenoic acid; EPA, eicosapentaenoic acid; $\gamma$-GTP, $\gamma$-glutamyl transpeptidase; GOT, glutamic-oxaloacetic transaminase; GPT, glutamic-pyruvic transaminase; BMI, body mass index. 
percentiles) of PFOS and PFOA levels in blood, serum levels of hepatic enzymes ( $\gamma$-GTP, GOT and GPT) and $\omega-3$ polyunsaturated fatty acids (DHA and EPA), BMI and frequency of intake of dishes, foods and alcoholic beverages. The medians of PFOS and PFOA levels in blood were 5.8 and $2.1 \mathrm{ng} \mathrm{m} l^{-1}$, respectively.

Tables 3 and 4 present adjusted mean blood concentrations of PFOS and PFOA according to sex, age category, regional block, residential area, smoking habit, reproductive history and method of nursing. Because of the positively skewed distributions even after log-transformation, adjusted mean concentrations of PFOS and PFOA were somewhat higher than the medians. Men showed significantly higher blood levels of PFOS than women. In both men and women, blood levels of PFOS increased with age, with statistically significant differences among five age categories. In women, levels of PFOA in blood also increased with age, and the difference among the age categories was significant. With regard to regional block, PFOS and PFOA levels were by far the highest in the Tokai/Hokuriku/Kinki region, where the adjusted means of PFOS and PFOA were significantly higher than those of all other regional blocks $(p<0.05$ by Tukey-Kramer's method). Concentrations of PFOS were highest in fishing villages, followed by urban areas and farming villages. However, there was no significant difference in PFOA levels in blood according to residential area. No significant difference was found in the blood levels of PFOS or PFOA according to smoking status. In women, those with experience of pregnancy had significantly lower PFOS and PFOA levels in blood. There was no difference in blood levels of PFOS or PFOA according to method of nursing.

Table 5 shows the correlation coefficients of PFOS and PFOA levels in blood with the frequency of intake of dishes, foods and alcoholic beverages and the serum levels of $\omega-3$ polyunsaturated fatty acids and liver enzymes. After adjustments for age, sex, BMI, regional block, smoking habits and other potential confounders (Model 2), frequency of intake of boiled fish in broth, sliced raw fish, and coastal fish showed significant positive correlations with PFOS, but not with PFOA. Some other dishes or food items were negatively correlated with blood levels of PFOS and PFOA. However, interpretation of the results was rather difficult. Serum levels of DHA and EPA had significant positive correlations with PFOS and PFOA. Serum levels GOT and GPT showed significant positive correlations with PFOS and PFOA in blood. On the other hand, frequencies of intake of beer, rice wine (sake) or spirits (syochu) were not significantly correlated with blood levels of PFOS or PFOA.

\section{Discussion}

In the present study, median PFOS and PFOA concentrations in whole blood were 5.8 and $2.1 \mathrm{ng} \mathrm{m} l^{-1}$, respectively. Most previous studies used serum or plasma as a biological medium to evaluate the body burden of PFOS and PFOA, and mean ratios of serum to whole blood concentrations were reported to be approximately 2.3 for PFOS and 2.0 for PFOA $^{16)}$. The estimated median serum level of PFOS in our study (13.3 $\left.\mathrm{ng} \mathrm{m} l^{-1}\right)$, using the ratio of 2.3, was similar to those in serum or plasma reported for Germany ${ }^{17)}$, Australia and Japan $\left(10.9-18.3 \mathrm{ng} \mathrm{m}^{-1}\right)^{18,19)}$, higher than those reported for Italy ${ }^{20)}$ Korea and Vietnam

Table 3. Adjusted mean concentrations of PFOS and PFOA in blood according to sex and age category

\begin{tabular}{|c|c|c|c|c|c|c|}
\hline & \multicolumn{3}{|c|}{ Men } & \multicolumn{3}{|c|}{ Women } \\
\hline & No. & $\begin{array}{c}\text { PFOS } \\
\text { Means }(\mathrm{ng} / \mathrm{m} l)\end{array}$ & $\begin{array}{c}\text { PFOA } \\
\text { Means }(\mathrm{ng} / \mathrm{m} l)\end{array}$ & No. & $\begin{array}{c}\text { PFOS } \\
\text { Means }(\mathrm{ng} / \mathrm{m} l)\end{array}$ & $\begin{array}{c}\text { PFOA } \\
\text { Means }(\mathrm{ng} / \mathrm{m} l)\end{array}$ \\
\hline Total & 307 & $6.8^{\mathrm{a}}$ & 2.4 & 298 & 4.9 & 2.4 \\
\hline$p$-value (sex) & & $<0.0001$ & 0.90 & & - & - \\
\hline \multicolumn{7}{|l|}{ Age category } \\
\hline $16-29$ & 39 & $5.5^{\mathrm{b}}$ & 2.4 & 32 & 3.3 & 2.2 \\
\hline $30-39$ & 75 & 6.1 & 2.4 & 59 & 3.2 & 2.0 \\
\hline $40-49$ & 78 & 6.1 & 2.4 & 63 & 3.9 & 2.5 \\
\hline $50-59$ & 74 & 7.5 & 2.4 & 76 & 5.9 & 2.6 \\
\hline $60-76$ & 41 & 8.6 & 2.3 & 68 & 11.5 & 4.3 \\
\hline$p$-value (age) & & $<0.0001$ & 0.96 & & $<0.0001$ & $<0.0001$ \\
\hline
\end{tabular}

${ }^{a}$ Adjusted for age, log (BMI), regional block, residential area, and smoking habits. ${ }^{\mathrm{b}}$ Adjusted for log (BMI), regional block, residential area, and smoking habits. PFOS, Perfluorooctanesulfonate; PFOA, perfluorooctanoate. 
Table 4. Adjusted mean concentrations of PFOS and PFOA in blood according to regional block, residential area, smoking habits, reproductive history and method of nursing

\begin{tabular}{|c|c|c|c|}
\hline & & PFOS & PFOA \\
\hline & No. & Means $(\mathrm{ng} / \mathrm{m} l)$ & Means $(\mathrm{ng} / \mathrm{m} l)$ \\
\hline \multicolumn{4}{|l|}{ Regional block ${ }^{\mathrm{a}}$} \\
\hline Hokkaido/Tohoku & 123 & 4.4 & 1.8 \\
\hline Kanto/Koshin-estu & 122 & 6.1 & 2.3 \\
\hline Tokai/Hokuriku/Kinki & 127 & 7.5 & 4.6 \\
\hline Chugoku/Shikoku & 120 & 6.0 & 2.2 \\
\hline Kyushu/Okinawa & 113 & 5.2 & 1.9 \\
\hline$p$-value & & $<0.0001$ & $<0.0001$ \\
\hline \multicolumn{4}{|l|}{ Residential area $^{\mathrm{a}}$} \\
\hline Urban & 266 & 5.3 & 2.3 \\
\hline Farming village & 135 & 5.0 & 2.5 \\
\hline Fishing village & 204 & 7.2 & 2.4 \\
\hline$p$-value & & $<0.0001$ & 0.48 \\
\hline \multicolumn{4}{|l|}{ Smoking habits ${ }^{\mathrm{a}}$} \\
\hline Nonsmoker & 382 & 6.0 & 2.3 \\
\hline Current smoker & 114 & 5.3 & 2.3 \\
\hline Ex-smoker & 109 & 5.9 & 2.6 \\
\hline Unknown & 3 & - & - \\
\hline$p$-value & & 0.12 & 0.16 \\
\hline \multicolumn{4}{|l|}{ Experience of pregnancy ${ }^{\mathrm{b}}$} \\
\hline Yes & 230 & 4.8 & 2.5 \\
\hline No & 66 & 7.2 & 3.6 \\
\hline Unknown & 2 & - & - \\
\hline$p$-value & & $<0.0001$ & $<0.0001$ \\
\hline \multicolumn{4}{|l|}{ Method of nursing ${ }^{\mathrm{b}}$} \\
\hline Breast & 63 & 6.1 & 2.7 \\
\hline Breast and bottle & 136 & 5.6 & 2.6 \\
\hline Bottle & 27 & 4.7 & 2.7 \\
\hline No delivery or unknown & 72 & - & - \\
\hline$p$-value & & 0.17 & 0.89 \\
\hline
\end{tabular}

a Adjusted for age, sex, log (BMI), regional block, residential area, and smoking habits, excluding the independent variable of interest. ${ }^{\mathrm{b}}$ Adjusted for age, log (BMI), regional block, residential area, and smoking habits. PFOS, Perfluorooctanesulfonate; PFOA, perfluorooctanoate.

(5.6-8.4 $\left.\mathrm{ng} \mathrm{m} l^{-1}\right)^{21,22)}$ and lower than those in the U.S. ${ }^{23,24)}$ and Denmark (30.2-35.8 $\left.\mathrm{ng} \mathrm{m}^{-1}\right)^{25)}$. On the other hand, the estimated median serum level of PFOA (4.2 $\mathrm{ng} \mathrm{m} l^{-1}$ ) was similar to those reported for Germany $^{17)}$, Denmark ${ }^{25)}$, the U.S. ${ }^{23,24)}$, Italy ${ }^{20)}$, Australia

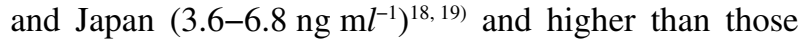
in Vietnam and Korea $\left(0.58-1.6 \mathrm{ng} \mathrm{ml^{-1 }}\right)^{21,22)}$.

With regard to gender, levels of PFOS but not PFOA in blood were significantly higher in men than in women. This result for PFOS was in line with those of other studies ${ }^{17,18,20,23,26)}$. One reason for this sex difference may be the effects of pregnancy. In our analysis in women, PFOS levels in blood were lower among those with an experience of pregnancy. One report showed that maternal serum levels of PFOS decreased significantly from 24-28 weeks of gestation to the time of delivery ${ }^{27)}$. Menstruation has been suggested as another route of excretion of $\mathrm{PFOS}^{28)}$. Although we could not evaluate this possibility because of a lack of data on menopausal status, high blood levels of PFOS in women aged 60 years or older were in accord with this notion. The effects of breast feeding seemed small, since there was no difference in PFOS levels in blood according to the 


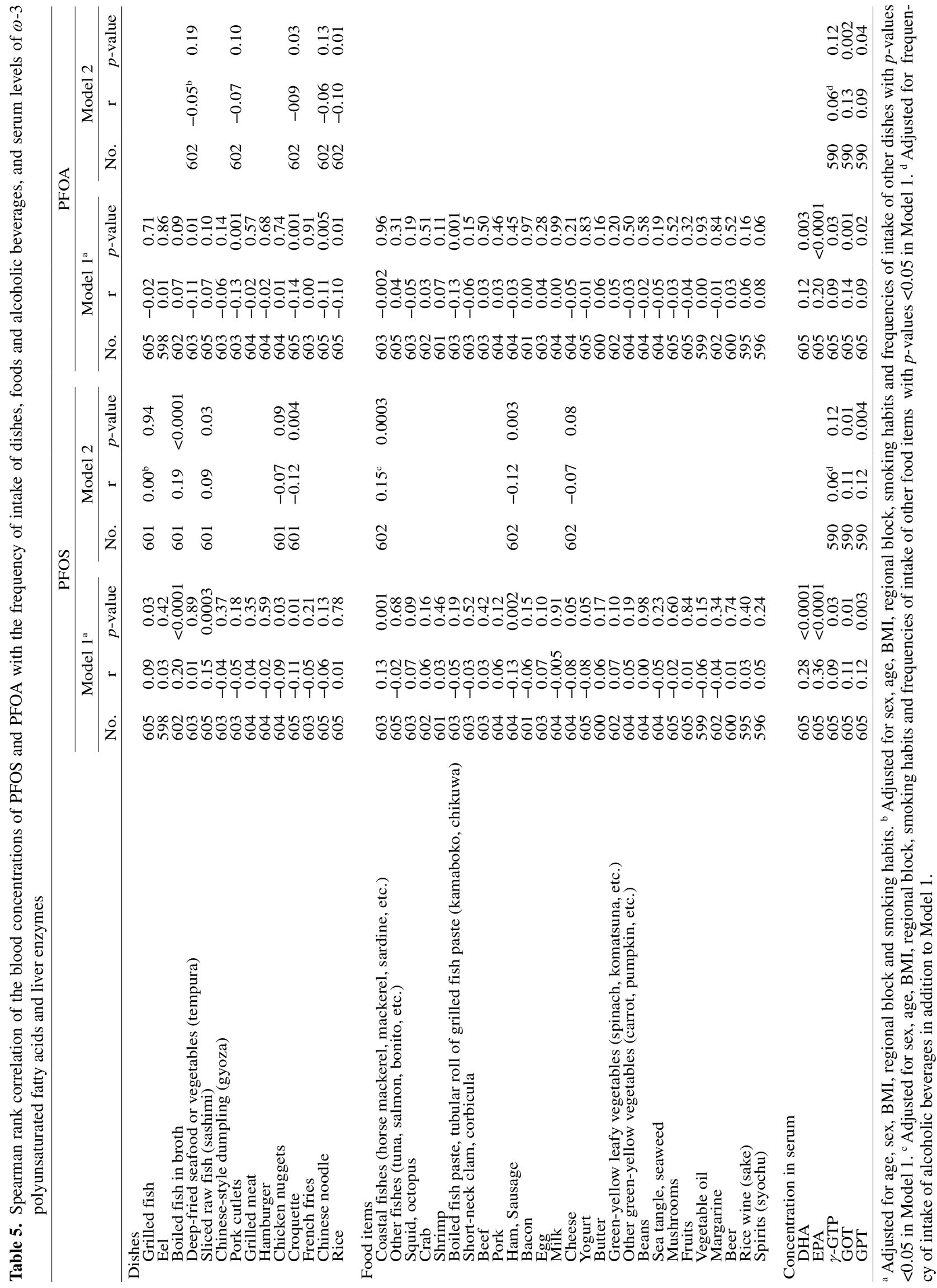


method of nursing. Other studies also showed that the milk/serum ratio of PFOS levels was approximately $0.01^{22,29)}$, suggesting the transfer of PFOS from mother's blood to breast milk to be rather small. Most of the earlier studies ${ }^{17,20,23,26)}$, but not others ${ }^{18,24)}$, showed significantly lower serum levels of PFOA in women than in men. The reason for the lack of sex difference in PFOA levels observed in our study may be in part because lower blood levels at reproductive ages (16-39 years) were canceled out by the higher blood levels at postmenopausal ages ( $\geq 60$ years) in women; it has been reported that blood levels of PFOA also decrease during pregnancy ${ }^{27)}$ and increase after menopause ${ }^{28)}$.

PFOS levels in blood increased with advancing age in both men and women, whereas PFOA levels increased with age only in women. The age-dependent increase in blood levels of PFOS and PFOA has been reported in earlier studies from Japan ${ }^{21)}$, Germany ${ }^{17)}$ and Italy $^{20)}$ but not in studies from the U.S. ${ }^{23,24)}$. The age-related increase in blood levels of these PFCs may be explained by the long biological half-lives and lower elimination rate after menopause in women. However, the reason for the lack of positive correlation between age and the levels of PFOA in men is unclear.

Regarding the relationship between blood levels of PFOS and smoking habits, the results from previous studies were inconsistent. Eriksen et al. ${ }^{25}$ reported that serum levels of PFOS were significantly lower in current smokers than never smokers and ex-smokers among Danish men. On the other hand, one Japanese study reported that there was no difference in PFOS levels in serum between nonsmokers and smokers ${ }^{26)}$. In our analysis, blood levels of PFOS were somewhat lower among current smokers than non- or ex-smokers, but the difference was not statistically significant.

Frequencies of intake of cooked fish and coastal fish, and serum levels of DHA and EPA, established biomarkers of fish intake ${ }^{30}$, were positively correlated with levels of PFOS in blood. Few studies have investigated the determinants of dietary intake or blood levels of PFOS in Japan. However, market basket studies have shown that dietary intake of PFOS derived mainly from fish and seafood in Spain ${ }^{10)}$, and cereals and cereal products, milk and dairy products, fish and seafood, and meat and meat products in Norway ${ }^{2}$. Two previous reports also reported that consumption of fish and shellfish was positively correlated with plasma or serum concentrations of PFOS in Norway ${ }^{9,31)}$. In contrast, meat, but not fish, was a significant predictor of PFOS levels in plasma among pregnant Danish women ${ }^{11}$. The high bioaccumulative potential of PFOS in the food web ${ }^{1)}$ and high intake of seafood in Japan, Spain and Norway may explain these results. The higher frequency of intake of fish may explain the higher blood levels of PFOS in fishing villages than urban areas and farming villages.

Levels of PFOS and PFOA in blood were found to be significantly correlated with the serum levels of hepatic enzymes. PFOS and PFOA are known to be hepatotoxic in rodents. Exposure of Sprague-Dawley rats to PFOS and PFOA at a dose of $20 \mathrm{mg} / \mathrm{kg}$ resulted in hepatomegaly and severe histological changes of the liver, such as fatty degeneration, hepatocytic necrosis, and inflammatory cell infiltration ${ }^{5)}$. Positive correlations of serum levels of PFOA with serum liver enzymes $(\gamma$-GTP, GOT or GPT) have been reported in several studies on fluorochemical workers with heavy exposure to PFOA or its related substance ${ }^{32,33)}$ but not in others ${ }^{34,35)}$. Positive correlations were also observed between serum levels of PFOA or PFOS and hepatic enzymes $(\gamma$-GTP and/or GPT) in populations of the U.S. with high or background exposure to these $\mathrm{PFCs}^{36,37)}$. One explanation for the positive associations found in the present study may be that liver cell damage alters the rate of elimination of PFOS and PFOA from the human body. Alternatively, background level exposure to PFOS and PFOA might have some adverse effects on liver function. Because this was a cross-sectional study, it was not possible to precisely discuss the cause-effect relationship.

Concentrations of PFOS and PFOA in blood were highest in the Tokai/Hokuriku/Kinki region. This regional difference persisted even after adjustments for age, sex, log (BMI), residential area, and smoking habits, and after exclusion of 91 subjects with liver dysfunction (serum GOT $\geq 40 \mathrm{IU} l^{-1}$ or GPT $\geq$ $35 \mathrm{IU} l^{-1}$, data not shown). If the subjects were limited to Kinki region (No. $=92$ ), the median (25th percentile, 75th percentile) levels of PFOS and PFOA were $10.5 \mathrm{ng} \mathrm{m} l^{-1}(6.7,16)$ and $6.8 \mathrm{ng} \mathrm{m}^{-1}(5.0,9.6)$, respectively. Harada et $a .^{26)}$ indicated that serum levels of PFOS and PFOA were significantly higher among subjects who had lived in the Kinki region for at least two years than other subjects. Another report also showed contamination of river water by these PFCs discharged from facilities such as a public water disposal site and an airport in the Kinki region ${ }^{38)}$. Concentrations of PFOS and PFOA in tap water were more than five times higher in the Kinki region than in the Tohoku region ${ }^{38)}$. Industries such as metal plating, firefighting, textile treatment, and semiconductor manufacturing have been considered as a source of environmental pollution by PFOS $^{39}$. Our results may support the notion that environmental contamination by PFOS and PFOA released from various industries account for the higher blood levels of these PFCs in the Kinki region.

Several limitations of the present study should be 
addressed. First, our questionnaire on the intake of foods and beverages queried the frequency but not portion size. However, it is generally recognized that most of the between-person variation in food intake is explained by the frequency of consumption, not portion size ${ }^{40)}$. Second, frequency of intake of foods and beverages over only the last month was asked, although dietary habit may be rather stable within the same person. Third, interindividual variation in the frequency of intake was small for several food items, as seen by the small interquartile ranges, which interfered with meaningful analysis of the association between dietary habits and blood levels of PFCs. Fourth, factors associated with blood levels of PFOS and PFOA were examined using a large number of items, which might have yielded spuriously significant results for some items. Fifth, because of the study design, subjects engaged in fishing and farming may have been oversampled compared with the general Japanese population $(0.3 \%$ for fishermen and $4.4 \%$ for farmers) (Statistical Bureau, Ministry of Public Management, Home Affairs, Posts and Telecommunications, 2005). Thus, mean blood levels of PFOS in the present study were considered to be somewhat higher than those of the general population. Finally, we did not examine the effects of other factors, such as drinking water and cookwares used.

In conclusion, our results suggest that blood levels of PFOS were mainly associated with consumption of fish. Blood levels of PFOS and PFOA were positively correlated with the serum levels of hepatic enzymes. Further investigations are needed to clarify the reason for the marked regional differences in the blood levels of PFOS and PFOA in Japan.

Acknowledgments: We are grateful to Drs. T. Kitamado, S. Morishita and N. Tsukamoto of the Ministry of the Environment and M. Hijiya and Y. Chisaki of Idea Consultants, Inc., for their cooperation and encouragement during the study. We also thank all members of the committee for the "Study on the Accumulation of Dioxins and Other Chemical Compounds in Humans", Drs. I. Uchiyama (Graduate School of Engineering, Kyoto University), F. Kayama (Jichi Medical School), H. Saito (Nagasaki University), T. Suzuki (The University of Tokyo, deceased), N. Suzuki (National Institute for Environment Studies), T. Sobue (National Cancer Center Research Institute), C. Tohyama (The University of Tokyo), S. Tominaga (Aichi Health Promotion Foundation), H. Miyata (Faculty of Pharmaceutical Science, Setsunan University), M. Morita (National Institute for Environment Studies / Ehime University), and S. Watanabe (National Institute of Health and Nutrition).

This study was performed under the supervision of the Ministry of the Environment of Japan. However, the views expressed in the present paper do not necessarily reflect the positions or policies of the ministry.

Conflict of interest: The authors declare that they have no conflicts of interest.

\section{References}

1) Butt CM, Berger Urs, Bossi R, Tomy Gregg $\mathrm{T}$. Levels and trends of poly-and perfluorinated compounds in the arctic environment. Sci Total Environ 2010; 408: 2936-65.

2) Haug LS, Salihovic S, Jogsten IE, et al. Levels in food and beverages and daily intake of perfluorinated compounds in Norway. Chemosphere 2010; 80: 1137-43.

3) Olsen GW, Burris JM, Ehresman DJ, et al. Half-life of serum elimination of perfluorooctanesulfonate, perfluorohexanesulfonate, and perfluorooctanoate in retiered fluorochemical production workers. Environ Health Perspect 2007; 115: 1298-305.

4) Johansson N, Fredriksson A, Eriksson P. Neonatal exposure to perfluorooctane sulfonate (PFOS) and perfluorooctanoic acid (PFOA) causes neurobehavioural defects in adult mice. Neurotoxicology 2008; 29: 160-9.

5) Cui L, Zhou Q, Liao C, Fu J, Jiang G. Studies on the toxicological effects of PFOA and PFOS on rats using histological observation and chemical analysis. Arch Environ Contam Toxicol 2009; 56: 338-49.

6) Genuis SJ, Birkholz D, Ralitsch M, Thibault N. Human detoxification of perfluorinated compounds. Public Health 2010; 124: 367-75.

7) Lindstrom AB, Strynar MJ, Libelo EL. Polyfluorinated compounds: past, present, and future. Environ Sci Technol 2011; 45: 7954-61.

8) Spliethoff HM, Tao L, Shaver SM, et al. Use of newborn screening program blood spots for exposure assessment: declining levels of perluorinated compounds in New York State infants. Environ Sci Technol 2008; 42: 5361-7.

9) Haug LS, Thomsen C, Brantsaeter AL, et al. Diet and particularly seafood are major sources of perfluorinated compounds in humans. Environ Int 2010; 36: $772-8$.

10) Ericson I, Martí-Cid R, Nadal M, Van Bavel B, Lindström G, Domingo JL. Human exposure to perfluorinated chemicals through the diet: intake of perfluorinated compounds in foods from the Catalan (Spain) market. J Agric Food Chem 2008; 56: 1787-94.

11) Halldorsson TI, Fei C, Olsen J, Lipworth L, McLaughlin JK, Olsen SF. Dietary predictors of perfluorinated chemicals: a study from the Danish National Birth Cohort. Environ Sci Technol 2008; 42: 8971-7.

12) Uemura H, Arisawa K, Hiyoshi M, et al. Prevalence of metabolic syndrome associated with body burden levels of dioxin and related compounds among 
Japan's general population. Environ Health Perspect 2009; 117: 568-73.

13) Arisawa K, Uemura H, Hiyoshi M, et al. Dietary patterns and blood levels of PCDDs, PCDFs, and dioxin-like PCBs in 1,656 Japanese individuals. Chemosphere 2011; 82: 656-62.

14) Hansen KJ, Clemen LA, Ellefson ME, Johnson HO. Compound-specific, quantitative characterization of organic fluorochemicals in biological matrices. Environ Sci Technol 2001; 35: 766-70.

15) SAS Institute. SAS/STAT Software: Changes and Enhancements through Release 6.12. SAS Institute, Cary 1997. 3.

16) Ehresman DJ, Froehlich JW, Olsen GW, Chang SC, Butenhoff JL. Comparison of human whole blood, plasma, and serum matrices for the determination of perfluorooctanesulfonate (PFOS), perfluorooctanoate (PFOA), and other fluorochemicals. Environ Res 2007; 103: 176-84.

17) Fromme $H$, Midasch $O$, Twardella $D$, Angerer $J$, Boehmer S, Liebl B. Occurrence of perfluorinated substances in an adult German population in southern Bavaria. Int Arch Occup Environ Health 2007; 80: 313-9.

18) Toms LM, Calafat AM, Kato K, et al. Polyfluoroalkyl chemicals in pooled blood serum from infants, children and adults in Australia. Environ Sci Technol 2009; 43: 4194-9.

19) Harada K, Koizumi A, Saito N, et al. Historical and geographical aspects of the increasing perfluorooctanoate and perfluorooctane sulfonate contamination in human serum in Japan. Chemosphere 2007; 66: 293-301.

20) Ingelido AM, Marra V, Abballe A, et al. Perfluorooctanesulfonate and perfluorooctanoic acid exposures of the Italian general population. Chemosphere 2010; 80: 1125-30.

21) Harada KH, Yang HR, Moon CS, et al. Levels of perfluorooctane sulfonate and perfluorooctanoic acid in female serum samples from Japan in 2008, Korea in 1994-2008 and Vietnam in 2007-2008. Chemosphere 2010; 79: 314-9.

22) Kim SK, Lee KT, Kang CS, et al. Distribution of perfluorochemicals between sera and milk from the same mothers and implications for prenatal and postnatal exposures. Environ Pollut 2011; 159: 169-74.

23) Olsen GW, Church TR, Miller JP, et al. Perfluorooctanesulfonate and other fluorochemicals in the serum of American Red Cross adult blood donors. Environ Health Perspect 2003; 111: 1892-901.

24) Olsen GW, Church TR, Larson EB, et al. Serum concentrations of perfluorooctanesulfonate and other fluorochemicals in an elderly population from Seattle, Washington. Chemosphere 2004; 54: 1599-611.

25) Eriksen KT, Sørensen M, McLaughlin JK, Tjønneland A, Overvad K, Raaschou-Nielsen O. Determinants of plasma PFOA and PFOS levels among 652 Danish men. Environ Sci Technol 2011; 45: 8137-43.

26) Harada $K$, Saito $N$, Inoue $K$, et al. The influence of time, sex and geographic factors on levels of perfluorooctane sulfonate and perfluorooctanoate in human serum over the last 25 years. J Occup Health 2004; 46: 141-7.

27) Monroy R, Morrison K, Teo K, et al. Serum levels of perfluoroalkyl compounds in human maternal and umbilical cord blood samples. Environ Res 2008; 108: 56-62.

28) Harada K, Inoue $K$, Morikawa A, Yoshinaga T, Saito N, Koizumi A. Renal clearance of perfluorooctane sulfonate and perfluorooctanoate in humans and their species-specific excretion. Environ Res 2005; 99: 253-61.

29) Kärrman A, Ericson I, van Bavel B, et al. Exposure of perfluorinated chemicals through lactation: levels of matched human milk and serum and a temporal trend, 1996-2004, in Sweden. Environ Health Perspect 2007; 115: 226-30.

30) Amiano P, Dorronsoro M, de Renobales M, Ruiz de Gordoa JC, Irigoien I, EPIC Group of Spain. Verylong-chain omega- 3 fatty acids as markers for habitual fish intake in a population consuming mainly lean fish: the EPIC cohort of Gipuzkoa. European Prospective Investigation into Cancer and Nutrition. Eur J Clin Nutr 2001; 55: 827-32.

31) Rylander C, Sandanger TM, Frøyland L, Lund E. Dietary patterns and plasma concentrations of perfluorinated compounds in 315 Norwegian women: the NOWAC Postgenome Study. Environ Sci Technol 2010; 44: 5225-32.

32) Olsen GW, Zobel LR. Assessment of lipid, hepatic, and thyroid parameters with serum perfluoroocatanoate (PFOA) concentrations in fluorochemical production workers. Int Arch Occup Environ Health 2007; 81: 231-46.

33) Sakr CJ, Leonard RC, Kreckmann KH, Slade MD, Cullen MR. Longitudinal study of serum lipids and liver enzymes in workers with occupational exposure to ammonium perfluoroactanoate. J Occup Environ Med 2007; 49: 872-9.

34) Olsen GW, Burris JM, Burlew MM, Mandel JH. Epidemiologic assessment of worker serum perfluoroocatanesulfonate (PFOS) and perfluorooctanoate (PFOA) concentrations and medical surveillance examinations. J Occup Environ Med 2003; 45: 260-70.

35) Costa G, Sartori S, Consonni D. Thity years of medical surveillance in perfluooctanoic acid production workers. J Occup Environ Med 2009; 51: 364-72.

36) Lin CY, Lin LY, Chiang CK, et al. Investigation of the associations between low-dose serum perfluorinated chemicals and liver enzymes in US adults. Am J Gastroenterol 2010; 105: 1354-63.

37) Gallo V, Leonardi G, Genser B, et al. Serum perfluorooctanoate (PFOA) and perfluorooctane sulfonate (PFOS) concentrations and liver function biomark- 
ers in a population with elevated PFOA exposure. Environ Health Perspect 2012; 120: 655-60.

38) Saito N, Harada K, Inoue $K$, Sasaki K, Yoshinaga T, Koizumi A. Perfluorooctanoate and perfluorooctane sulfonate concentrations in surface water in Japan. J Occup Health 2004; 46: 49-59.
39) Xie S, Wang T, Liu S, et al. Industrial source identification and emission estimation of perfluorooctane sulfonate in China. Environ Int 2013; 52: 1-8.

40) Willett WC. Nutritional Epidemiology, second edition. New York (United States): Oxford University Press; 1998. p.101-47. 